This item is the archived peer-reviewed author-version of:

\title{
Identifying panic disorder subtypes using factor mixture modeling
}

\section{Reference:}

Pattyn Thomas, Van Den Eede Filip, Lamers Femke, Veltman Dick, Sabbe Bernard, Penninx Brenda W..- Identifying panic disorder subtypes using factor mixture modeling

Depression and anxiety - ISSN 1091-4269 - 32:7(2015), p. 509-517

Full text (Publishers DOI): http://dx.doi.org/doi:10.1002/da.22379

To cite this reference: http://hdl.handle.net/10067/1253980151162165141 


\section{WILEY \\ Online Proofing System}

1. Corrections should be marked with the Adobe Annotation \& Comment Tools below:

PowerProof: eProof window - Internet Explorer
Complete Proof Review $\quad$ (3) Help Note:To access the "Complete Proof Review" button, please access the Proof PDF through link provided in the mail.

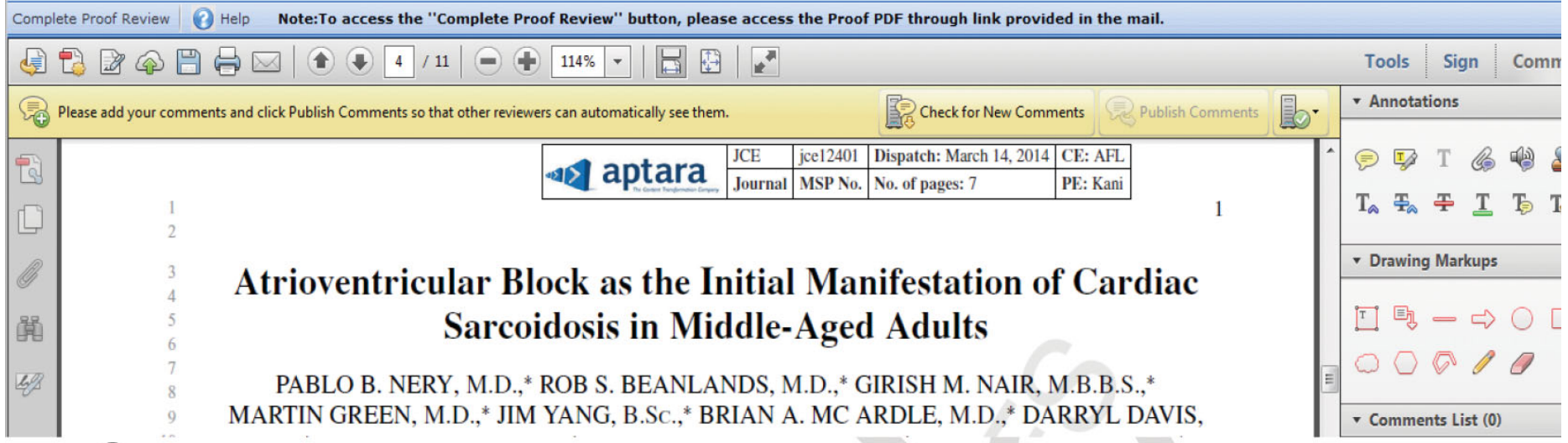

2. To save your proof corrections, click the 'Publish Comments' button. Publishing your comments saves the marked up version of your proof to a centralized location in Wiley's Online Proofing System. Corrections don't have to be marked in one sitting - you can publish corrections and log back in at a later time to add more.

3. When your proof review is complete we recommend you download a copy of your annotated proof for reference in any future correspondence concerning the article before publication. You can do this by clicking on the icon to the right of the 'Publish Comments' button and selecting 'Save as Archive Copy...'.

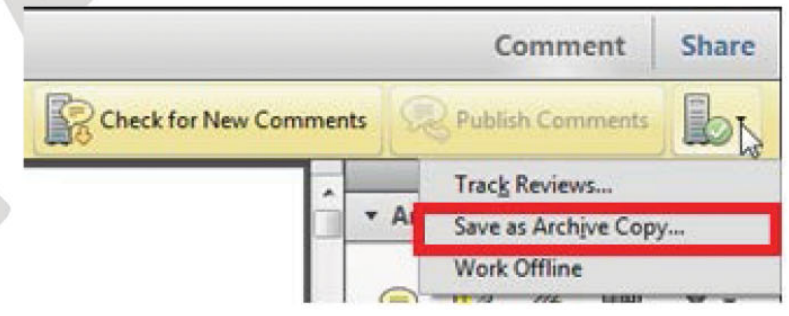

4. When your proof review is complete and you are ready to send corrections to the publisher click the 'Complete Proof Review' button that appears above the proof in your web browser window. Do not click the 'Complete Proof Review' button without replying to any author queries found on the last page of your proof. Incomplete proof reviews will cause Complete Proof Review a delay in publication. Note: Once you click 'Complete Proof Review' you will not be able to mark any further comments or corrections.

\section{Firefox, Chrome, Safari Users}

If your PDF article proof opens in any PDF viewer other than Adobe Reader or Adobe Acrobat, you will not be able to mark corrections and query responses, nor save them. To mark and save corrections, please follow these instructions to disable the built-in browser PDF viewers in Firefox, Chrome, and Safari so the PDF article proof opens in Adobe within a Firefox or Chrome browser window. 
Required software to e-Annotate PDFs: Adobe Acrobat Professional or Adobe Reader (version 9.0 or above). (Note that this document uses screenshots from Adobe Reader $\mathbf{X}$ )

The latest version of Acrobat Reader can be downloaded for free at: http://get.adobe.com/reader/

Once you have Acrobat Reader open on your computer, click on the Comment tab at the right of the toolbar:

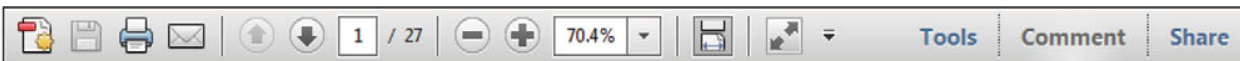

This will open up a panel down the right side of the document. The majority of tools you will use for annotating your proof will be in the Annotations section, pictured opposite. We've picked out some of these tools below:

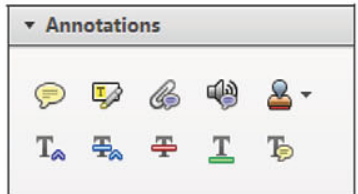

1. Replace (Ins) Tool - for replacing text.

Th Strikes a line through text and opens up a text box where replacement text can be entered.

\section{How to use it}

$\otimes$ Highlight a word or sentence.

$\triangle$ Click on the Replace (Ins) icon in the Annotations section.

$\otimes \quad$ Type the replacement text into the blue box that appears.

Idard tramework for the analysis of $\mathrm{m}$ icy Nevertheless, it also-led to exog،

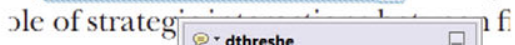
\begin{tabular}{l|l|l|l|l|l} 
& $\Theta$ dthreshe \\
aber of comp & $08 / 06 / 2011$ 15:58:17
\end{tabular}

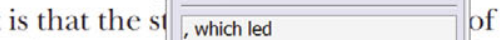
nain compo: be level, are exc nc important wores ox cres on wir M henceforth) 1 we snen the 'hlarl b

3. Add note to text Tool - for highlighting a section to be changed to bold or italic.

T) Highlights text in yellow and opens up a text box where comments can be entered.

\section{How to use it}

$\otimes$ Highlight the relevant section of text.

$\otimes$ Click on the Add note to text icon in the Annotations section.

\ype instruction on what should be changed regarding the text into the yellow box that annears

namic responses of mark ups ent with the VAR evidence

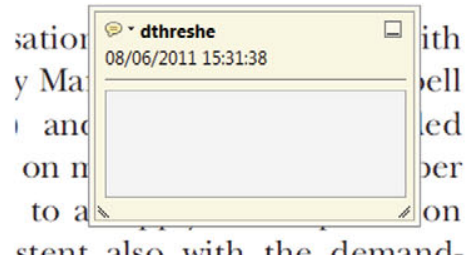

2. Strikethrough (Del) Tool - for deleting text

Strikes a red line through text that is to be deleted.

How to use it

\ Highlight a word or sentence.

$\bowtie$ Click on the Strikethrough (Del) icon in the Annotations section.

there is no room for extra prohts as s ups are zero and the number of iet) values are not determined by Blanchard and Kiyotaki (1987), sfect competition in general equilil ts of aggregate demand and supply lassical framework assuming mono oon on ovoronous numbor of firmo

4. Add sticky note Tool - for making notes at specific points in the text.

Marks a point in the proof where a comment needs to be highlighted.

How to use it

$\otimes$ Click on the Add sticky note icon in the Annotations section.

$\otimes$ Click at the point in the proof where the comment should be inserted.

$\otimes \quad$ Type the comment into the yellow box that appears.

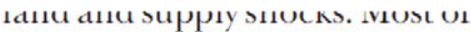

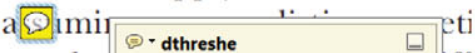
numbe 08/06/2011 15:18:08 iff

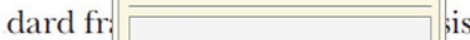
cy. Nev , )le of stu wi

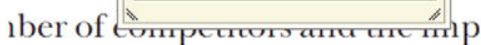
is that the structure of the secto 
5. Attach File Tool - for inserting large amounts of text or replacement figures.

Inserts an icon linking to the attached file in the appropriate pace in the text.

\section{How to use it}

- Click on the Attach File icon in the Annotations section.

- Click on the proof to where you'd like the attached file to be linked.

- Select the file to be attached from your computer or network.

- Select the colour and type of icon that will appear in the proof. Click OK.

E N D

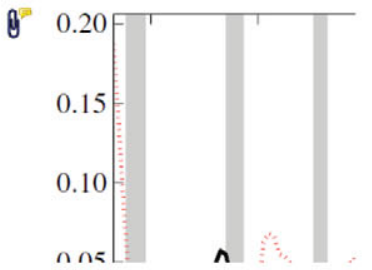

6. Add stamp Tool - for approving a proof if no corrections are required.

Inserts a selected stamp onto an appropriate place in the proof.

How to use it

- Click on the Add stamp icon in the Annotations section.

- Select the stamp you want to use. (The Approved stamp is usually available directly in the menu that appears).

- Click on the proof where you'd like the stamp to appear. (Where a proof is to be approved as it is, this would normally be on the first page).

It the Dusiness cycie, starting with the on perfect competition, constant ret

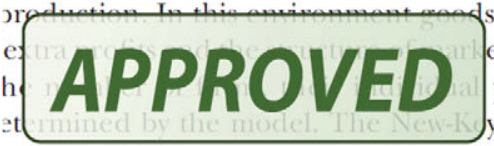
otaki (1987), has introduced produc general equilibrium models with nomin

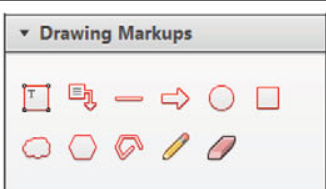

How to use it

- Click on one of the shapes in the Drawing Markups section.

- Click on the proof at the relevant point and draw the selected shape with the cursor.

- To add a comment to the drawn shape, move the cursor over the shape until an arrowhead appears.

- Double click on the shape and type any text in the red box that appears.
7. Drawing Markups Tools - for drawing shapes, lines and freeform annotations on proofs and commenting on these marks.

Allows shapes, lines and freeform annotations to be drawn on proofs and for comment to be made on these marks.

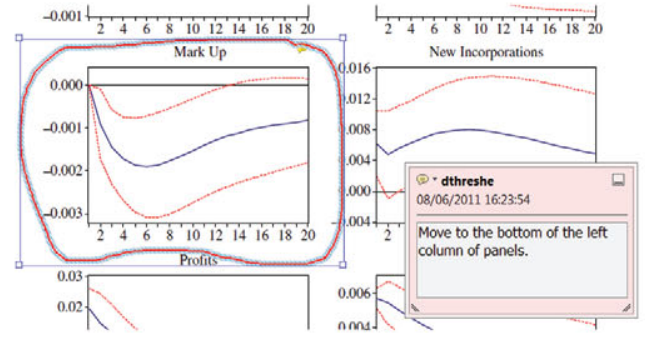

For further information on how to annotate proofs, click on the Help menu to reveal a list of further options:

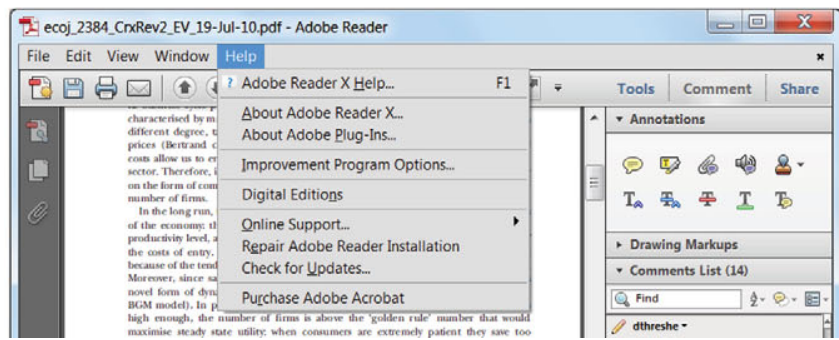




\title{
Research Article
}

\section{IDENTIFYING PANIC DISORDER SUBTYPES USING FACTOR MIXTURE MODELING}

\author{
Thomas Pattyn, M.D., ${ }^{1,2 *}$ Filip Van Den Eede, ${ }^{2,1}$ Femke Lamers, ${ }^{3,4}$ Dick Veltman, ${ }^{4}$ Bernard G. Sabbe, ${ }^{1,5}$
} and Brenda W. Penninx ${ }^{4}$

\begin{abstract}
Background: The clinical presentation of panic disorder (PD) is known to be bighly beterogeneous, complicating research on its etiology, neurobiological pathways, and treatment. None of the attempts to identify PD subtypes bave been independently reproduced, rendering the current literature inconclusive. Methods: Using a data-driven, case-centered approach (factor mixture modeling) on a broad range of anxiety symptoms assessed with the Beck anxiety inventory, the present study identifies PD disorder subtypes in a large $(n=658)$, welldocumented mixed-population sample from the Netherlands Study of Depression and Anxiety (NESDA), with subtypes being validated and detailed using a variety of clinical characteristics. Results: $A$ three-class, one-factor model proved superior to all other possible models (Bayesian information criterion = 13,200; Lo-Mendel-Rubin $=0.0295$; bootstrapped likelibood ratio test $\leq 0.0001)$, with the first class, a cognitive-autonomic subtype, accounting for $29.8 \%$, the second class, the autonomic subtype, for $29.9 \%$, and a third class, the aspecific subtype, for $40.3 \%$ of the population. The cognitive-autonomic and autonomic subtypes showed significant differences compared to the aspecific subtype (e.g., comorbidity and suicide attempts) but on severity differed between themselves only. Conclusion: Three qualitatively different PD subtypes were identified: a severe cognitive-autonomic subtype, a moderate autonomic subtype, and a mild aspecific subtype. Qualitative and quantitative differences were related to severity and clinical properties such as comorbidity, suicide attempts, sleep, and sense of mastery. Depression and Anxiety 0:1-10, 2015. (을 2015 Wiley Periodicals, Inc.
\end{abstract}

\begin{abstract}
${ }^{1}$ Collaborative Antwerp Psychiatric Research Institute (CAPRI), University of Antwerp, Antwerp, Belgium

2Department of Psychiatry, Antwerp University Hospital, Antwerp, Belgium

${ }^{3}$ National Institute of Mental Health, Bethesda, USA

${ }^{4}$ Department of Psychiatry, EMGO Institute of Health and Care Research, Neuroscience Campus Amsterdam, VU University Medical Center, Amsterdam, The Netherlands

${ }^{5}$ Department of Psychiatry, Psychiatric Hospital Duffel, Duffel, Belgium
\end{abstract}

Contract grant sponsor: Geestkracht program of the Netherlands Organization for Health Research and Development (Zon-Mw); Contract grant number: 10-000-1002; Contract grant sponsor: VU University Medical Center; Contract grant sponsor: GGZ inGeest; Contract grant sponsor: Arkin; Contract grant sponsor: Leiden University Medical Center; Contract grant sponsor: GGZ Rivierduinen; Contract grant sponsor: University Medical Center Groningen; Contract grant sponsor: Lentis, Contract grant sponsor: GGZ Friesland;
Contract grant sponsor: GGZ Drenthe; Contract grant sponsor: Scientific Institute for Quality of Healthcare (IQ healthcare); Contract grant sponsor: The Netherlands Institute for Health Services Research (NIVEL); Contract grant sponsor: The Netherlands Institute of Mental Health and Addiction (Trimbos); Contract grant sponsor: Cassiers bequest for research on panic disorder (CAPRI).

*Correspondence to: Thomas Pattyn, Collaborative Antwerp Psychiatric Research Institute (CAPRI), University of Antwerp, Universiteitsplein 1 R3.22, 2610 Antwerp, Belgium.

E-mail: thomas.pattyn@uantwerpen.be

Received for publication 30 October 2014; Revised 5 February 2015; Accepted 21 April 2015

DOI 10.1002/da.22379

Published online in Wiley Online Library

(wileyonlinelibrary.com). 


\begin{abstract}
Key words: anxiety disorder; classification; factor analysis; latent class analysis; panic; factor mixture modeling; subtype; Beck anxiety inventory; NESDA; nosology
\end{abstract}

\author{
Abbreviations \\ $\mathrm{PD}=$ panic disorder \\ $\mathrm{EFA}=$ exploratory factor analysis \\ LCA $=$ latent class analysis \\ $\mathrm{FMM}=$ factor mixture modeling \\ $\mathrm{BAI}=$ Beck anxiety inventory \\ ASI $=$ anxiety sensitivity index \\ $\mathrm{FEAR}=$ fear questionnaire \\ WHODAS $=$ World Health Organization Disability \\ Assessment Schedule
}

\section{INTRODUCTION}

In the DSM-IV and DSM-5, the most recent version, panic disorder (PD) is defined as having recurrent and unexpected panic attacks or episodes of intense fear or discomfort with at least four of 13 additional symptoms, ${ }^{[1,2]}$ thus covering a broad variety of clinical presentations. PD is also known to have a high lifetime and current comorbidity with psychiatric disorders such as depression and other anxiety disorders. ${ }^{[3]}$ Furthermore, the literature shows substantial inconsistencies in neurobiological findings for PD, e.g., with regard to HPA-axis functioning, ${ }^{[4]}$ electrophysiological activity, ${ }^{[5]}$ regional brain morphology, and neurotransmitter receptor density. ${ }^{[6]}$ All of the above indicates that the pathogenesis and manifestations of PD are likely to be far more heterogeneous than originally assumed, which inherently complicates treatment but also clinical research.

To date, theoretical research has frequently focused on respiratory and cognitive aspects of PD. Major theoretical models of panic are the cognitive theories of Beck, Clark and Bandura, ${ }^{[7-10]}$ and Klein's false suffocation alarm. ${ }^{[1]}$ A key feature of cognitive models is cognitive misinterpretation, such as catastrophic thinking, that occurs in response to a normal physiological sensation. In contrast, in Klein's model, a biologically erroneous stimulus, for example an abnormal CO2 sensitivity, prompts a cognitively correct interpretation. ${ }^{[10]}$ More recently, a context-sensitivity panic-vulnerability model. ${ }^{[12]}$ has been proposed as an explanatory model for the propensity to experience panic in situations that pose no objective threat to the individual (cf., cognitive misinterpretation or Klein's false suffocation alarm model).

Employing a more data-driven, variable-centered approach by applying a factor analysis to 14 DSM-III$\mathrm{R}$ panic symptoms to identify $\mathrm{PD}$ subtypes in a clinical sample, Briggs et al. ${ }^{[13]}$ concluded that, in a twodimensional model, the presence of respiratory items or their absence was the most distinguishing criterion. More recently, Roberson-Nay and Kendler. ${ }^{[14]}$ adopted exploratory factor analysis (EFA), latent class analysis (LCA), and factor mixture modeling (FMM), the latter two both case-centered approaches, to identify possible subtypes in different samples mostly using DSM-III and DSM-III-R panic symptoms. Results revealed at least two subtypes in all samples, with the strongest subtype discriminator being high or low loadings on respiratory items, prompting the authors to propose a respiratory and a nonrespiratory subtype with a possible third mild respiratory subtype. ${ }^{[14]}$

The aim of the present study was to investigate PD subtypes in a large and well-documented, contemporary, mixed-population sample using EFA, LCA, and FMM on a broad range of panic symptoms derived from the 21 items of the Beck anxiety inventory (BAI). Second, we aimed to validate these subtypes using other anxiety indicators and to identify distinctive demographic and clinical characteristics for the various subtypes.

\section{MATERIALS AND METHODS}

\section{SAMPLE}

Data were derived from the Netherlands Study of Depression and Anxiety (NESDA), an ongoing multisite naturalistic cohort study. The sample comprises 2,981 participants aged between 18 and 65 years who were recruited between September 2004 and February 2007 from the general community, general (family) practices, and mental health facilities to ensure the representation of depression and anxiety psychopathology in various developmental stages and different treatment settings. Detailed descriptions of NESDA's design can be found elsewhere. ${ }^{[15]}$ Psychiatric diagnoses (DSM-IV) were made using the Composite International Diagnostic Interview, version 2.1. ${ }^{[16]}$ Although a key feature of $\mathrm{PD}$, panic attacks occur episodically, varying from daily to once a month, while for a sound PD diagnosis, patients must also be presenting with a persistent concern or change in behavior for at least one month. Thus, to ensure inclusion of all types of patients, we selected 670 patients with a current baseline diagnosis of PD with or without agoraphobia with a 6-month recency criterion. Twelve patients were excluded because of incomplete information on anxiety symptoms, resulting in a final sample of 658 participants.

\section{BECK ANXIETY INVENTORY}

As it was our objective to include a broad range of panic-related anxiety symptoms in our analysis, we opted for the BAI, a 21-item selfreport instrument that assesses the overall severity of anxiety ${ }^{[17]}$ and is designed to improve discrimination between depression and anxiety. Respondents are asked to rate how much they have been bothered by each symptom in the last week on a 4 -point Likert scale $(1=$ not at all; $2=$ mildly; $3=$ moderately; $4=$ severely). The items were dichotomously coded as absent $=1 / 2$ or present $=3 / 4$. We used all 21 items for the EFA, LCA, and FMM. All DSM items, excluding chest pain and derealization, were well represented in the BAI, with some being represented by two or more BAI items (DSM: trembling, BAI: hands trembling/wobbliness in legs). The BAI additionally includes five items not represented in the DSM-IV PD criteria: being unable to 
relax, feeling terrified, nervous, scared, and fear of worst happening. The reliability and validity of the BAI have been well established in general populations and in a psychiatric sample ${ }^{[17-19]}$.

\section{VALIDATION AND DESCRIPTION OF IDENTIFIED SUBTYPES}

Four groups of variables were evaluated to validate the identified PD subtypes and to detail subtype-specific features:

Demographics. Age, sex, and educational level (in years).

Anxiety Indicators. The anxiety sensitivity index (ASI) ${ }^{[20]}$ assessing the fear of anxiety-related sensations, the Fear Questionnaire (FEAR) ${ }^{[21]}$ assessing the severity of phobic avoidance, and two validated BAI subscales ${ }^{[22]}$ : a 14-item somatic $(1-3,6-8,11-13,15,18-21)$ and a 7-item subjective subscale $(4,5,9,10,14,16,17)$.

Clinical Variables. Comorbidity, that is, current comorbid affective disorders (major depressive disorder, social phobia, general anxiety disorder), number of suicide attempts, frequent use of antidepressants, benzodiazepine usage (at least $50 \%$ of the time), the WHODAS functioning score (World Health Organization Disability Assessment Schedule II $)^{[23]}$ as an overall disability index, the mastery scale score ${ }^{[24]}$ indicating the extent to which a person perceives himself to be in control of events or situations, and the three subscale scores of the Mood and Anxiety Symptom Questionnaire-30, ${ }^{[25]}$ with the negative affect subscale gauging emotional states such as fear, anger, or guilt; the positive affect subscale assessing moods such as feeling active, excited, delighted, enthusiastic, interested; and the somatic arousal subscale evaluating physiological arousal symptoms such as trembling, shaking, dizziness, sweating, and heart racing, having some overlap with BAI items.

Physical Health Indicators. Current smoking; sleep (insomnia rating scale scores) ${ }^{[26]}$; physical activity as assessed with the International Physical Activity Questionnaire ${ }^{[27]}$ and expressed per 1,000 metabolic equivalent $\mathrm{min} /$ week; the number of somatic diseases for which treatment is being received including respiratory disease, osteoarthritis, cancer, gastrointestinal disease, liver disease, epilepsy, thyroid disease, diabetes, and cardiovascular disease.

\section{STATISTICAL ANALYSIS}

To identify PD subtypes we used FMM, a statistical method that combines principles of EFA and LCA. ${ }^{[28]}$ While EFA is a variablecentered approach that models the data in a set of latent continuous factors, LCA uses a case-centered approach that allows latent categorical factors to be identified and assigns cases to these classes. FMM allows latent categorical classes (subtypes) to be identified while considering a continuous factor (dimensionality) in one integrated model. A more comprehensive explanation of these techniques can be found elsewhere. ${ }^{[14],[28-33]}$ In the current study, we adopted the Mplus 5.1 software $^{[29]}$ for all three methods.

Our stepwise analysis started with EFA to examine panic symptom dimensionality and factor structures (Fig. 1, step 1). Statistical interpretation was inferred from a combination of the Kaiser criterion (eigenvalues $>1$ ), qualitative interpretation of factor loadings, and a screeplot. A limitation of EFA is its assumption of homogeneity, which may not be met in a PD population. In the second step, LCAs were conducted to examine possible categorical panic classes. An important limitation of this technique is its inability to integrate dimensionality (e.g., panic severity) in its model (Fig. 1, step 2). Therefore, in the third and final step we ran a series of FMMs, integrating the principles of EFA and LCA, to examine all class-factor combinations withheld with EFA and LCA, with FMM model types ranging from restrictive models to models of increasing flexibility (Fig. 1, step 3).

In the first FMM type, the full invariant type, item threshold, and factor loadings were class invariant, factor variance was set to be equal across classes, and factor means fixed to zero. Two partially invariant FMM types were examined; one was defined with class noninvariant factor loadings and factor variance (the factor-invariant type) and the second defined by class noninvariant thresholds (the thresholdinvariant type). A fourth, noninvariant, type was examined, where all parameters were class noninvariant and allowed to be freely estimated (see Fig. 1, step 3). LCA and FMM results were evaluated by modelfit statistics, designating how well the data statistically fits the model, such as the Bayesian information criterion (lower is preferred), ${ }^{[30,31]}$ Lo-Mendell-Rubin's chi-square difference test $\left(<0.05\right.$ is preferred), ${ }^{[32]}$ the bootstrapped likelihood ratio test $\left(\leq 0.05\right.$ is preferred), ${ }^{[33]}$ and the theoretical interpretability of the model.

Subtypes were described using SPSS 20 on demographics, anxiety indicators, clinical variables, and physical health indices. Appropriate parametric tests (chi-square for categorical variables, analysis of variance for continuous variables) and nonparametric tests (KruskalWallis) were performed. Post hoc analyses were adjusted by means of Bonferroni corrections (analysis of variance) and Dunn test (KruskalWallis) at a significance level of 0.05 .

\section{RESULTS}

\section{SAMPLE}

Our final sample included $658 \mathrm{PD}$ patients with a mean age of 41 years, of whom $70.5 \%$ was female.

\section{EXPLORATORY FACTOR ANALYSIS}

Following the Kaiser criterion, a maximum of five factors could be extracted from the data (see Supplementary Tables, online). Further investigation using the screeplot technique and qualitative interpretation of the factor loadings showed high factor loadings on all BAI items when a single factor was extracted, suggesting a severity dimensionality. When two or more factors were extracted, qualitatively distinct factors emerged, suggesting distinct categorical classes and indicating the irrelevance of a multifactorial structure. Importantly, between-item correlations may be due to mean differences between possible classes, meaning that observed factor structures may not directly translate into classes. Nonetheless, up to three factors were included in the FMM analysis.

\section{LATENT CLASS ANALYSIS}

A four-class model showed the best model-fit statistics (Bayesian information criterion $=13,500$; Lo-MendelRubin $=0.0001$; bootstrapped likelihood ratio test $\leq 0.0001$ ) (Table 1, upper section). A five-class model yielded a better (lower) Bayesian information criterion value of 13,475 and a similar bootstrapped likelihood ratio test $(<0.0001)$ but the $P$-value of the Lo-MendellRubin was no longer significant $(=0.44)$, indicating that the five-class model did not improve model fit. Interpretation of the four-class model revealed two qualitatively distinct classes and two comparable classes but with lower item probabilities, merely delineating a severe and less severe category within these two qualitatively distinct classes. Up to four classes were included in the FMM. 


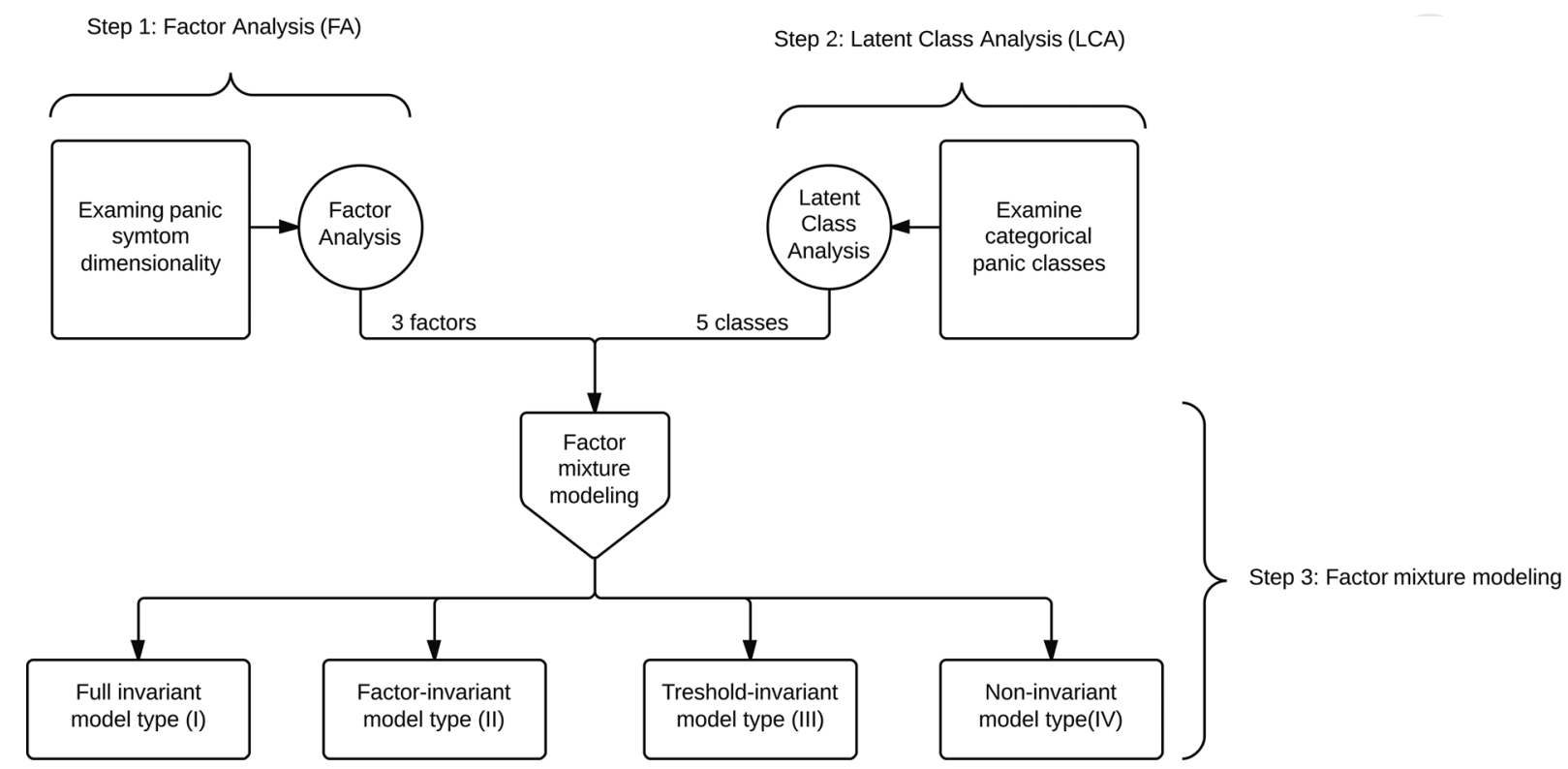

Figure 1. Overview of analytical strategy.

\section{FACTOR MIXTURE MODELING}

FMM models up to five classes and three factors were examined, each with four different model types as explained above (one invariant, two semi-invariant, and one noninvariant type). All multifactorial models yielded poorer model-fit statistics than unifactorial models. Results from the unifactorial, factor-invariant, multiple classes models are presented in Table 1, lower section.

A three-class, one-factor model yielded the best fit (Bayesian information criterion $=13,200$; Lo-MendelRubin $=0.0295$; bootstrapped likelihood ratio test $\leq 0.0001)$. The results listed in Fig. 2 were therefore taken from the three-class, one-factor model, which was overall the best fitting data-driven solution. In the figure, the item probabilities for the three-class one-factor model are graphically depicted. Mean posterior probabilities for most likely class membership were 0.895 for class $1,0.830$ for class 2 , and 0.836 for class 3 , indicating a good classification quality. Class 1 accounted for $29.8 \%(n=196)$ of the sample and had the highest item probabilities on almost all items but was mainly differentiated from the other two classes by having very high item probabilities on cognitive items such as being scared, terrified, fear of losing control, fear of dying,

TABLE 1. Latent class and factor mixture fit indices

\begin{tabular}{|c|c|c|c|c|c|c|}
\hline Number of classes & Pars & BIC & $\begin{array}{l}\text { ss analysis } \\
\text { LMR }\end{array}$ & VLMR & BLRT & $\log 1$ \\
\hline 1 & 21 & 15904.960 & / & / & / & -7884.343 \\
\hline 2 & 43 & 13874.255 & 0.0000 & 0.0000 & 0.0000 & -6797.610 \\
\hline 3 & 65 & 13582.977 & 0.0000 & 0.0000 & 0.0000 & -6580.589 \\
\hline 4 & 87 & 13500.513 & 0.0001 & 0.0001 & 0.0000 & -6467.976 \\
\hline 5 & 109 & 13475.329 & 0.4375 & 0.4341 & 0.0000 & -6384.003 \\
\hline \multicolumn{7}{|c|}{ Partially invariant factor mixture model ${ }^{\mathrm{a}}$} \\
\hline Number of classes & Pars & BIC & LMR & VLMR & BLRT & Logl \\
\hline 1 & 43 & 13382.183 & / & / & / & -6551.574 \\
\hline 2 & 67 & 13243.409 & 0.0099 & 0.0096 & 0.0000 & -6404.316 \\
\hline 3 & 91 & 13200.570 & 0.0295 & 0.0289 & 0.0000 & -6305.026 \\
\hline 4 & 115 & 13249.323 & 0.5012 & 0.4988 & 0.0000 & -6251.532 \\
\hline 5 & NC & $\mathrm{NC}$ & NC & $\mathrm{NC}$ & $\mathrm{NC}$ & NC \\
\hline
\end{tabular}

BIC, Bayesian information criterion; V(LMR), (Voung) Lo-Mendell-Rubin likelihood ratio test; BLRT, bootstrap likelihood ratio test; NC, not calculated because earlier defined models were found to be nonsignificant.

Lower BIC values indicate better fit. (V)LMR and BTLR $P$-values $<0.05$ are acceptable.

${ }^{\text {a }}$ Partially invariant unifactorial model; factor loadings constrained to be equal across classes, thresholds are free; factor means fixed at zero in the first group and free in others. 


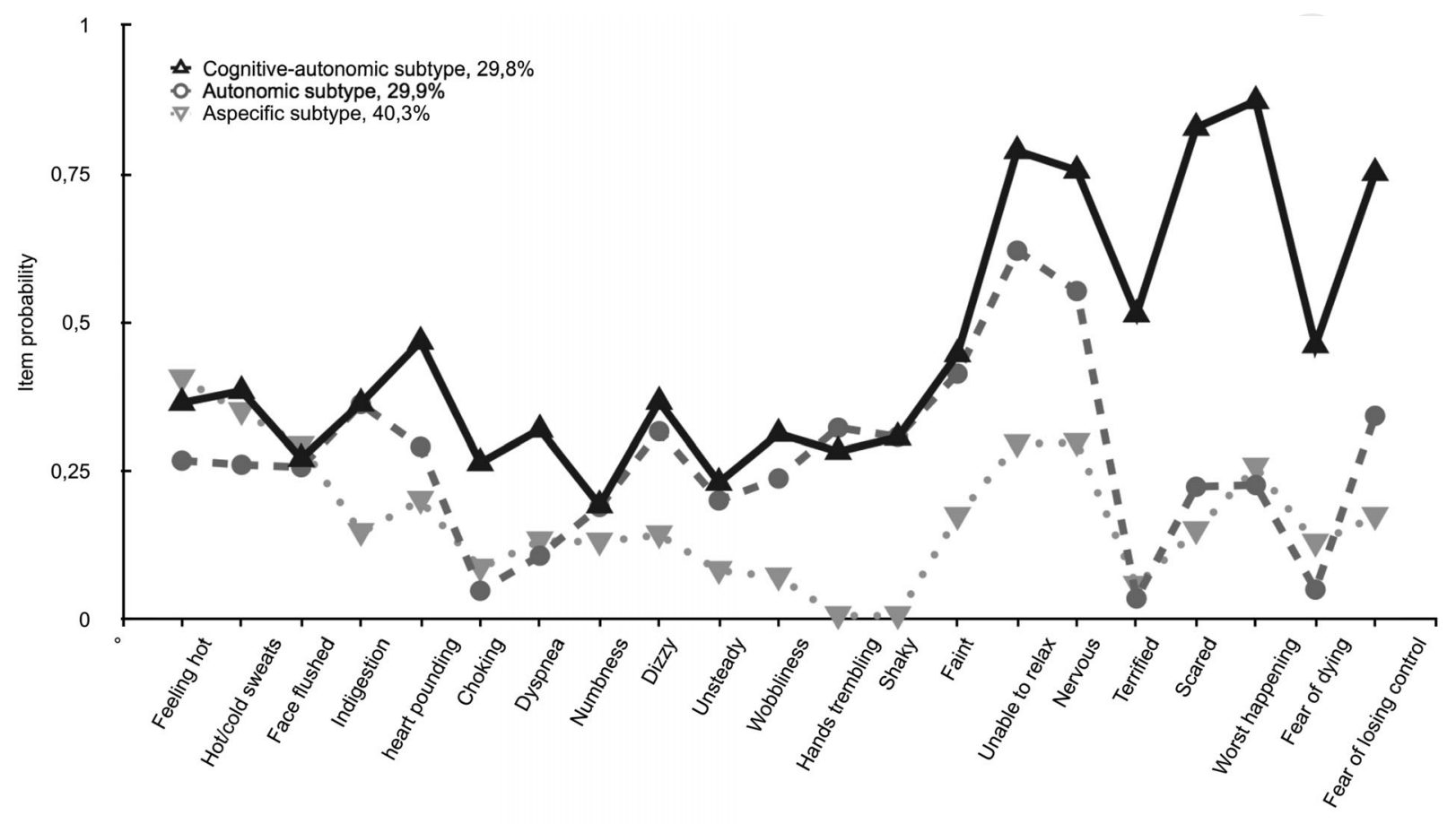

Beck Anxiety Inventory item

Figure 2. Three-class one-factor mixture model.

and fear of the worst happening. We therefore labeled this class "the cognitive-autonomic subtype". Class 2 accounted for $29.9 \%(n=197)$ and was characterized by the lowest probabilities on respiratory items such as feeling of choking and difficulty breathing while also having low probabilities on cognitive items but similar levels of autonomic item probabilities (e.g., feeling hot, indigestion, and face flushed) as class 1 and was therefore termed "the autonomic subtype". Accounting for $40.3 \%$ $(n=265)$ of the sample, class 3 was the largest class, had overall low item probabilities and was mainly differentiated from the other two classes by having low autonomic and neurological item probabilities on items such as indigestion, numbness, hands/legs trembling, feeling shaky, and dizziness.

\section{DESCRIPTION OF SUBTYPES}

Demographics, anxiety indicators, and clinical characteristics are shown in Tables 2 and 3. Classes did not differ as to age, sex, or educational level. Examination of other additional anxiety indicators showed a clear order among the classes, where class 1 scored higher than class 2 on every indicator, with class 3 having the lowest scores on all anxiety indicators, indicating that classes differed on anxiety-severity indices as well as on panicsymptom dimensions. Post hoc analysis showed that total BAI scores differed statistically and clinically among all three subtypes, with class 1 being classified as severe (>25), class 2 as moderate (between 16 and 25), and class 1 as mild (between 8 and 15), while on the somatic sub- scale class 1 and class 2 only differed from class 3 but not from each other. Highest anxiety sensitivity and fear of phobias was found in class 1 with statistically significantly lower scores for class 2 and the lowest scores for class 3 consistent with item probabilities for cognitive items.

All classes also revealed a high current psychiatric comorbidity. Class 1 differed from the two other classes by having the highest current agoraphobia rates $(70.9 \%$ vs. 59.9 and $60.8 \%$, respectively). Class 3 distinguished itself from the other two classes with lower scores on all three Mood and Anxiety Symptom Questionnaire scales, better functioning, fewer suicide attempts, and a higher sense of mastery. The use of benzodiazepines was significantly greater in classes 1 and 2 compared to class 3 , but no between-class differences were found in the use of antidepressants. However, the prevalence of current comorbid depressive disorder was significantly higher in classes 1 and 2 compared to class 3 (Table 3 ).

With regard to physical health indices, no differences were found for current smoking, physical activity, and number of somatic diseases. Only the subjective sleep index signaled significantly more sleeping problems (insomnia rating scale $>8$ ) in classes 1 and 2 relative to class 3.

\section{DISCUSSION}

The present study sought to identify subtypes of panic disorder in a large patient sample using factor mixture techniques allowing the identification of 
TABLE 2. Demographic characteristics and anxiety indicators

\begin{tabular}{|c|c|c|c|c|}
\hline & $\begin{array}{c}\text { Class } 1(n=196) \\
\text { cognitive- } \\
\text { autonomic } \\
\text { D }\end{array}$ & $\begin{array}{c}\text { Class } 2(n=197) \\
\text { autonomic } \\
\text { c characteristics }\end{array}$ & $\begin{array}{c}\text { Class } 3(n=265) \\
\text { aspecific }\end{array}$ & $\begin{array}{l}\text { Overall } \\
P \text {-value }\end{array}$ \\
\hline Age, mean (SD, year) & $39.43(11.94)$ & $41.12(12.22)$ & $41.95(11.46)$ & 0.760 \\
\hline Female & $71 \%$ & $68 \%$ & $73 \%$ & 0.510 \\
\hline Education, mean (SD, year) & $11.19(3.41)$ & $11.24(3.44)$ & $11.60(3.12)$ & 0.340 \\
\hline Anxiety indices & & & & \\
\hline BAI total score, mean (SD) & $29.30(9.80)$ & $20.97(7.76)$ & $15.41(11.31)$ & $<0.001^{\mathrm{a}, \mathrm{b}, \mathrm{c}}$ \\
\hline Somatic subscale, mean (SD) & $15.73(7.49)$ & $13.52(6.05)$ & $9.56(7.52)$ & $<0.001^{\mathrm{a}, \mathrm{b}}$ \\
\hline Subjective subscale, mean (SD) & $13.57(3.29)$ & $7.45(2.77)$ & $5.85(4.32)$ & $<0.001^{\mathrm{a}, \mathrm{b}, \mathrm{c}}$ \\
\hline ASI, mean (SD) & $41.75(10.66)$ & $37.20(9.08)$ & $33.96(9.99)$ & $<0.001^{\mathrm{a}, \mathrm{b}, \mathrm{c}}$ \\
\hline FEAR, mean (SD) & $45.54(22.01)$ & $39.25(20.24)$ & $32.68(20.46)$ & $<0.001^{\mathrm{a}, \mathrm{b}, \mathrm{c}}$ \\
\hline
\end{tabular}

BAI, Beck anxiety inventory; ASI, anxiety sensitivity index; SD, standard deviation.

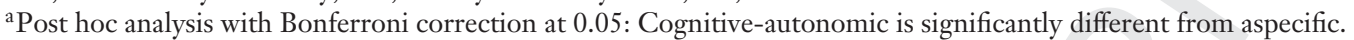

${ }^{\mathrm{b}}$ Post hoc analysis with Bonferroni correction at 0.05 : Autonomic is significantly different from aspecific.

${ }^{c}$ Post hoc analysis with Bonferroni correction at 0.05: Cognitive-autonomic is significantly different from autonomic.

categorical classes while incorporating dimensionality in an integrated model with BAI scores as input.

Based on our data, we propose three symptom-based PD subtypes: a "cognitive-autonomic" subtype with a $29.8 \%$ prevalence, an "autonomic" subtype with a $29.9 \%$ prevalence, and an "aspecific" subtype with a $40.3 \%$ prevalence. We found additional quantitative differences among the subtypes mainly in the severity dimension, with the cognitive-autonomic subtype being the most and the aspecific subtype the least severe. The cognitive-autonomic and autonomic subtypes mostly

TABLE 3. Clinical characteristics

\begin{tabular}{|c|c|c|c|c|}
\hline & $\begin{array}{c}\text { Class } 1(n=196) \\
\text { cognitive- } \\
\text { autonomic } \\
\text { Psychiatric ce }\end{array}$ & $\begin{array}{l}\text { Class } 2(n=197) \\
\quad \text { autonomic } \\
\text { idity }\end{array}$ & $\begin{array}{c}\text { Class } 3(n=265) \\
\text { aspecific }\end{array}$ & $\begin{array}{l}\text { Overall } \\
P \text {-value }\end{array}$ \\
\hline Current comorbid anxiety disorder & $67.3 \%$ & $64.0 \%$ & $47.2 \%$ & $<0.001^{\mathrm{a}, \mathrm{b}}$ \\
\hline Social phobia & $57.1 \%$ & $48.7 \%$ & $35.8 \%$ & $<0.001^{\mathrm{a}, \mathrm{b}}$ \\
\hline Generalized anxiety disorder & $33.2 \%$ & $32.5 \%$ & $23.4 \%$ & $0.033^{\mathrm{a}, \mathrm{b}}$ \\
\hline PD with agoraphobia & $70.9 \%$ & $59.9 \%$ & $60.8 \%$ & $0.037^{\mathrm{a}, \mathrm{b}}$ \\
\hline $\begin{array}{l}\text { Current comorbid depressive disorder } \\
\text { Clinical characteristics }\end{array}$ & $66.8 \%$ & $63.5 \%$ & $50.6 \%$ & $0.001^{\mathrm{a}, \mathrm{b}}$ \\
\hline \multicolumn{5}{|l|}{ Clinical characteristics } \\
\hline Mastery scale, mean (SD) & $13.87(4.32)$ & $14.63(4.10)$ & $16.42(3.81)$ & $<0.001^{\mathrm{a}, \mathrm{b}}$ \\
\hline WHODAS, mean (SD) & $47.07(21.03)$ & $45.83(20.33)$ & $30.49(22.81)$ & $<0.001^{\mathrm{a}, \mathrm{b}}$ \\
\hline \multicolumn{5}{|l|}{ MASQ } \\
\hline Somatic arousal, mean (SD) & $22.89(6.67)$ & $21.63(6.46)$ & $17.86(6.78)$ & $<0.001^{\mathrm{a}, \mathrm{b}}$ \\
\hline Negative affect, mean (SD) & $27.56(8.95)$ & $25.55(8.11)$ & $20.93(7.97)$ & $<0.001^{\mathrm{a}, \mathrm{b}}$ \\
\hline Positive affect, mean (SD) & $39.45(7.99)$ & $39.25(7.55)$ & $35.09(9.27)$ & $<0.001^{\mathrm{a}, \mathrm{b}}$ \\
\hline Number of serious suicide attempts, mean (SD) & $0.47(1.41)$ & $0.46(0.96)$ & $0.27(0.87)$ & $0.052^{\mathrm{a}}$ \\
\hline Current use of benzodiazepines & $37.8 \%$ & $37.1 \%$ & $23.8 \%$ & $0.001^{\mathrm{a}, \mathrm{b}}$ \\
\hline Current use of antidepressants & $48.5 \%$ & $49.7 \%$ & $41.9 \%$ & 0.184 \\
\hline \multicolumn{5}{|l|}{ Physical health indicators } \\
\hline Current smoker & $51.5 \%$ & $54.3 \%$ & $47.2 \%$ & 0.750 \\
\hline Sleep (insomnia rating scale $>8$ ) & $67.5 \%$ & $61.7 \%$ & $51.5 \%$ & $0.005^{\mathrm{a}, \mathrm{b}}$ \\
\hline Number of chronic somatic diseases, mean (SD) & $0.95(1.08)$ & $1.13(1.17)$ & $1.02(1.08)$ & 0.295 \\
\hline Physical activity (MET/week), mean (SD) & $3,407(2,781)$ & $3,800(3,139)$ & $3,674(3,348)$ & 0.466 \\
\hline
\end{tabular}

PD, panic disorder; MET, metabolic equivalent minutes; MASQ, Mood and Anxiety Symptom Questionnaire; WHODAS, World Health Organization Disability Assessment Schedule II; SD, standard deviation.

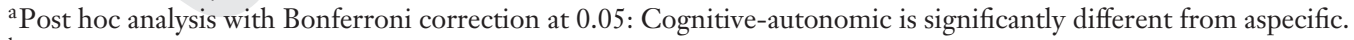

${ }^{b}$ Post hoc analysis with Bonferroni correction at 0.05 : Autonomic is significantly different from aspecific.

${ }^{c}$ Post hoc analysis with Bonferroni correction at 0.05: Cognitive-autonomic is significantly different from autonomic. 
showed significant differences in anxiety sensitivity and phobic avoidance (as assessed with ASI and FEAR).

Our results are in line with some, but not all, earlier efforts to identify PD subtypes. Adopting a factorial approach, Meuret et al. ${ }^{[34]}$ concluded that panic symptom dimensions have an impact on the severity and mastery of anxiety symptoms and proposed a three-factor solution consisting of a cardiorespiratory, a mixed somatic, and a cognitive dimension. These dimensions coincide with two of our subtypes, where the cardiorespiratory and cognitive dimensions are most strongly represented in the cognitive-autonomic subtype and the mixed somatic dimension in the autonomic subtype. However, the third category we identified was characterized by the absence of any clear dimensions and hence labeled the aspecific subtype.

Using a similar approach but with different results, Roberson-Nay and Kendler (2011) concluded that in all but one of their samples, a two-class one-factor model was preferable. This discrepancy may be explained by differences among cohorts: a treatment-seeking sample, a twin sample, and three epidemiological samples in the Robertson-Nay and Kendler study ${ }^{[14]}$ and a patient sample recruited from the general population, family practices, and outpatient clinics in ours. Also, earlier diagnoses were based on DSM-III/DSM-III-R criteria rather than the DSM- IV criteria in our study, with definitions differing mainly as to the "required" number and frequency of panic attacks. Theoretically, these disparities may have led to the exclusion of patients with a particular PD type, which would then explain Robertson-Nay and Kendler's two-class solution. ${ }^{[14]}$ Additionally, applying DSM-IV criteria, the authors also found a one-factor three-class model with a severe respiratory, a mild respiratory, and a nonrespiratory subtype.

Respiratory aspects of PD have attracted ample attention from researchers largely owing to Klein's false suffocation alarm theory and the widespread use of carbon dioxide inhalation to induce panic attacks in laboratory settings. ${ }^{[11,35]}$ Arguably, this field of research tends to view cognitive symptoms as secondary phenomena, ${ }^{[36]}$ potentially creating a bias toward respiratory subtyping. Roberson-Nay and Kendler ${ }^{[14]}$ based their definition of respiratory subtypes on the high loadings on cardiorespiratory symptoms and high endorsements of all DSM items, whereas the more cognitive subtype was characterized by high levels of anxiety but low endorsement on all DSM items. In contrast and showing the highest endorsement on all DSM items, our first class was characterized by prominent cardiorespiratory symptoms but equally so by high anxiety levels. Our findings thus indicate that the extent to which respiratory and cognitive subtypes differ may be limited, whereas our distinction between autonomic and aspecific subtypes may have more clinical relevance. Nevertheless, additional longitudinal research on the course and stability of the proposed subtypes is clearly necessary to establish their clinical usefulness.
Evaluating whether the three classes showed substantial differences on demographic features, anxiety scores, clinical variables, and physical health indices, we mostly found differences between classes 1 and 2 relative to class 3, with the exception of the ASI and FEAR total scores that also differed between class 1 and class 2, where ASI indicates anxiety sensitivity and FEAR total scores phobic avoidance. This is an interesting finding considering Telch et al.'s context-sensitivity vulnerability model, $[12,38]$ where anxiety sensitivity is an important and possibly predictive measure of panic attacks induced by internal or external contextual cues. Class 3 was associated with lower psychiatric comorbidity, fewer suicide attempts, lower insomnia and WHODAS ratings, and less use of benzodiazepines than classes 1 and 2. Besides qualitative differences on symptomatology, the subtypes were also significantly different on anxiety indicators and clinical characteristics, differences that could not be explained by any demographic dissimilarities.

Following the proposed cut-off values, ${ }^{[18,36]}$ the BAI total scores for each subtype were also clinically significant, with class 1 classifying as severe $(>25)$, class 2 as moderate (16-25), and class 3 as mild (815).Comorbidity profiles also characterized classes 1 and 2 , while showing that class 3 may represent a less severe clinical entity. Interestingly, the cognitive-autonomic subtype had the highest depressive comorbidity but the lowest use of antidepressants, for which we have no obvious explanation. The above mainly illustrates a severity dimension, where the first class "the cognitiveautonomic subtype" is the most severe, the second class "the autonomic subtype" a more moderate, and the third class "the aspecific subtype" a mild subtype.

Additionally, classes 1 and 2 had differential scores on the BAI subjective subscale, the ASI, and FEAR total scores but not on the BAI somatic subscale or the Mood and Anxiety Symptom Questionnaire subscales. This is consistent with the qualitative differences we obtained for the two subtypes on the cognitive items. Item probabilities for all cognitive items were also highest in class 1 and lowest in class 3 , in accordance with differential ASI and FEAR total scores, signifying that the level of anxiety sensitivity and/or phobic avoidance may be an important factor in the development of cognitive symptoms and more severe panic symptoms. Also, there were no between-subtype differences on the mastery scale, suggesting that the ASI might be a better predictor than the mastery scale. However, as we computed the highest mastery scale score for class 3 , it is plausible to suggest that a higher sense of mastery influences the clinical presentation, which would be consistent with class 3 having the lowest scores for the BAI subjective subscale, ASI, and FEAR.

This paper provides clear evidence that FMM can be effectively used to explore possible subtypes in psychiatric research. While FMM principles are generally known, advantages and disadvantages of the technique need to be considered for a proper interpretation of the results. Besides using a robust statistical method to 
evaluate the computed FMM models as to classification criteria and probability scores, the decision on the final best-fit model should take the interpretability of the model into account. While this allows for current theories to be included, thereby enhancing the clinical usefulness of the results, it also implies that choosing the final model is to some extent subjective. However, in our comprehensive analyses, both the statistical results and interpretability clearly pointed to the same three-class one-factor model.

Our study does have some other limitations that warrant discussion. While trained interviewers made the PD diagnoses, the BAI-from which we derived the symptom ratings-is a self-report questionnaire, which may partly explain differences with earlier studies. Moreover, the BAI was developed by a cognitive psychiatrist, ${ }^{[18]}$ which may have introduced a bias in the nature and number of (cognitive) items. Also, the BAI was not originally designed to measure panic but rather anxiety in general. However, the literature shows that the scale is more closely aligned to PD than to any other anxiety disorder, with higher BAI scores in panic populations than in nonpanic anxiety populations, ${ }^{[39-42]}$ while its items capture the DSM-criteria for PD best. Previous studies using DSM panic symptom criteria for statistical analysis were limited by their retrospective assessment of specific symptoms that only briefly occur during a panic attack (seconds to minutes) that may have gone largely unnoticed at the time of the attack because other symptoms were predominant. Also, in view of the current discussions about the boundaries between psychiatric disorders, it can be argued that DSM-IV panic symptoms may be too rigid or arbitrary, which is supported by the newly formulated DSM-5 cultural-specific panic attack criteria such as headache, tinnitus, etc. A more extended questionnaire like the BAI may overcome these limitations. Unfortunately, the BAI does not gauge certain panic features such as anticipated panic, perceived panic consequences, or perceived inability to effectively cope with panic attacks. Future research should also consider these features.

In order to validate the proposed PD subtypes and substantiate the clinical relevance of subtyping in general, future research should investigate their longitudinal stability, that is, whether the subtypes are consistent throughout the course of the disorder and its treatment. Also, the symptomatically different subtypes should be tested for their potentially different neurobiological pathways, etiologies, and treatment approaches. Additional genetic information, neuroanatomical, neurophysiological, and cognitive variables on possible subtypes are crucial and should, when becoming available, be included in the subtyping scheme to validate the subtypes further and to improve the clinical benefits of the subtypes. Furthermore, a comparable methodology should be applied to other nonpanic anxiety groups (e.g., general anxiety disorder and social phobia), to explore whether similar subtyping is viable in these groups.
In conclusion, using factor mixture analysis, a datadriven case-centered approach that permits dimensionality, we found a three-class one-factor model to be superior to all other models, enabling us to identify three qualitatively distinct panic disorder subtypes that showed significant quantitative differences on anxiety sensitivity, phobic avoidance, psychiatric comorbidity, suicide attempts, and other clinical characteristics. Based on their quantitative and qualitative differences, we propose the following three PD subtypes: a severe cognitiveautonomic subtype (29.8\%), a moderate autonomic subtype (29.9\%), and a mild aspecific subtype (40.3\%).

Acknowledgments. We would like to thank the reviewers for their much valued time and comments, and Mrs. Meulenbroek for her linguistic suggestions.

Conflict of interest. The authors report no financial or other relationships relevant to the subject of the article.

\section{REFERENCES}

1. American Psychiatric Association. Diagnostic and Statistical Manual of Mental Health Disorders: DSM IV. 4th ed. Washington, DC: American Psychiatric Association; 1994.

2. American Psychiatric Association. Diagnostic and Statistical Manual of Mental Health Disorders: DSM 5. 5th ed. Washington, DC: American Psychiatric Association; 2013.

3. Kessler RC, Chiu WT, Demler O, Walters EE. Prevalence, severity and comorbidity of 12 -month DSM-IV disorders in the National Comorbidity Survey Replication. Arch Gen Psychiatry 2005;62:617-627.

4. Abelson JL, Khan S, Liberzon I, Young EA. HPA axis activity in patients with panic disorder: review and synthesis of four studies. Depress Anxiety 2007;24:66-76.

5. Clark RC, Galletly CA, Ash DJ, et al. Evidence-based medicine evaluation of electrophysiological studies of the anxiety disorders. Clinical EEG Neurosci 2009;40:84-112.

6. Roy-Burne PR, Craske MG, Stein MB. Panic disorder. Lancet 2006;368:1023-32.

7. Beck AT, Emery G, Greenberg RL. Anxiety Disorders and Phobias: A Cognitive Perspective. New York, NY: Basic books; 1985.

8. Clark DM. A cognitive model of panic. Behav Res Ther 1986;24:461-470.

9. Bandura A. Self-efficacy conception of anxiety. Anxiety Res 1988;1:77-98

10. Fava L, Morton J. Causal modeling of panic disorder theories. Clin Psychol Rev 2009;29:623-637.

11. Klein DF. False suffocation alarms, spontaneous panics and related conditions. An integrative hypothesis. Arch Gen Psychiatry 1993;50:306-317.

12. Telch JM, Smits JAJ, Brown M, et al. Effect of threat context and cardiac sensitivity on fear responding to a $35 \% \mathrm{CO} 2$ challenge: a test of the context-sensitivity panic vulnerability model. J Behav Ther Exp Psychiatry 2010;41:365-372

13. Briggs AC, Stretch DD, Brandon S. Subtyping the panic disorder by symptom profile. Br J Psychiatry 1993;163:201-209.

14. Roberson-Nay R, Kendler KS. Panic disorder and its subtypes: a comprehensive analysis of panic symptom heterogeneity using epidemiological and treatment seeking samples. Psychol Med 2011;41:2411-2421. 
15. Penninx B, Beekman A, Smit J, et al. The Netherlands Study of Depression and Anxiety (NESDA): rationale, objectives and methods. Int J Methods Psychiatr Res 2008;17(3):121-140.

16. World Health Organization. Composite International Diagnostic Interview, Core Version 2.1: Interviewer's Manual. Sydney, Australia.

17. Beck AT, Epstein N, Brown G, Steer RA. An inventory for measuring clinical anxiety: psychometric properties. J Consult Clin Psychol 1988;56:893-897.

18. Beck AT, Steer AR. Manual for the Beck Anxiety Inventory. San Antonio, TX: Psychological Corporation; 1993.

19. Osman A, Hoffman J, Kopper FXBBA, et al. Factor structure, reliability and validity of the Beck anxiety inventory in adolescent psychiatric inpatients. J Clin Psychol 2002;58:443-456.

20. Reiss S, Peterson RA, Gursky DM, McNally RJ. Anxiety sensitivity, anxiety frequency and the prediction of fearfulness. Behav Res Ther 1986;24:1-8.

21. Marks IM, Mathews AM. Brief standard self-rating for phobic patients. Behav Res Ther 1979;17:263-267.

22. Kabacoff RI, Segal DL, Hersen M, van Hasselt VB. Psychometric properties and diagnostic utility of the Beck anxiety inventory and the state-trait anxiety inventory with older adult psychiatric outpatients. J Anxiety Disord 1997;11:33-47.

23. Garin O, Ayuso-Mateos JL, Almansa J, et al. Validation of the "World Health Organization Disability Assessment Schedule, WHODAS-2" in patients with chronic diseases. Health Qual Life Outcomes 2010;8:51.

24. Pearlin LI, Schooler C. The structure of coping. J Health Social Behav 1978;19:2-21.

25. Wardenaar KJ, van Veen T, Giltay EJ, et al. Development and validation of a short version of the mood and anxiety symptoms questionnaire: the MASQ-30. Psychiatry Res 2010;179:101-106.

26. Levine DW, Lewis MA, Bowen DJ, et al. Reliability and validity of the Women's Health Initiative Insomnia Rating Scale. Psychol Assess 2003;15:137-148.

27. Ainsworth BE, Bassett DR, Strath SJ, et al. Comparison of three methods for measuring the time spent in physical activity. Med Sci Sports Exerc 2000;32:457-64.

28. Lubke GH, Muthen B. Investigating population heterogeneity with factor mixture models. Psychol Methods 2005;10(1):21-39

29. Muthen LK, Muthen BO. Mplus User's Guide. 5th ed. Los Angeles, CA: Muthen \& Muthen; 2007.
30. Schwarz G. Estimating the dimensions of a model. Ann Statist 1978;6:461-464.

31. Nylund KL, Asparouhov T, Muthen BO. Deciding on the number of classes in latent class analysis and growth mixture modeling: a monte carlo simulation study. Struct Equ Modeling 2007; 14:535569.

32. Lo Y, Mendell NR, Rubin DB. Testing the number of components in a normal mixture. Biometrika 2001;88:77-778.

33. McLachlan GJ. On bootstrapping the likelihood ratio test statistic for the number of components in a normal mixture. Appl Stat 1978;23:318-324.

34. Meuret AE, White KS, Ritz T, et al. Panic attack symptom dimensions and their relationship to illness characteristics in panic disorder. J Psychiatr Res 2006;40:520-527.

35. Rassovsky Y, Kusher MG. Carbon dioxide in the study of panic disorder: issues of definition, methodology and outcome.J Anxiety Disord 2003;17:1-32.

36. Rush AJ, First MB, Burns B. Handbook of Psychiatric Measures, 2nd ed., Washington, DC: American Psychiatric Publishing; 2008.

37. Sinha S, Papp LA, Gorman JM. How study of respiratory physiology aided our understanding of abnormal brain function in panic disorder. J Affect Disord 2000;61(3):191-200.

38. Telch JM, Harrington PJ, Smits JAJ, Powers MB. Unexpected arousal, anxiety sensitivity, and their interaction on CO2-induced panic: further evidence for the context-sensitivity vulnerability model. J Anxiety Disord 2011;25:6454-653.

39. De Beurs E, Wilson KA, Chambless DL, Goldstein AJ, Feske U. Convergent and divergent validity of the Beck anxiety inventory for patients with panic disorder and agoraphobia. Depress Anxiety 1997;6(4):140-146.

40. Cox BJ, Cohen E, Direnfeld DM, Swinson RP. Does the Beck Anxiety Inventory measure anything beyong panic attack symptoms? Behav Res Ther 1996;34(11-12):949-954

41. Leyger OT, Ruberg HL, Woodruff-Borden J. Examination of the utility of the Beck anxiety inventory and its factors as a screener for anxiety disorders. J Anxiety Disord 2006;20(4): $444-58$

42. Muntigh AD, Van der Feltz-Cornelis CM, Van Marwijk HW, Spinhoven P, Penninx BW, Van Balkom AJ. Is the Beck Anxiety Inventory a good tool to assess the severity of anxiety? A primary care study in the Netherlands Study of Depression and Anxiety (NESDA). BMC Fam Pract 2011;4:12. 


\section{Author Query Form}

\begin{tabular}{ll}
\hline Journal & DA \\
Article & da22379
\end{tabular}

Dear Author

During the copy-editing of your paper, the following queries arose. Please respond to these by marking up your proofs with the necessary changes/additions. Please write your answers clearly on the query sheet if there is insufficient space on the page proofs. If returning the proof by fax do not write too close to the paper's edge. Please remember that illegible mark-ups may delay publication.

\begin{tabular}{|c|c|c|}
\hline Query No. & Description & Remarks \\
\hline Q1 & $\begin{array}{l}\text { Author: Please confirm that given names (red) and surnames/family names (green) have been iden- } \\
\text { tified correctly. }\end{array}$ & \\
\hline Q2 & Wiley: Please check the abbreviation list as typeset for correctness. & \\
\hline Q3 & Author: Please provide the academic degrees for all the authors. & \\
\hline Q4 & Author: Please provide department name for affiliation 3, if available. & \\
\hline Q5 & Author: Please check affiliations 2 and 5 as typeset for correctness. & \\
\hline Q6 & Author: Please check the grant information as typeset for correctness. & \\
\hline Q7 & Author: Please define "BLRT" in Table 1. & \\
\hline$\overline{Q 8}$ & Author: Please check the term "Logl" in Table 1 as typeset for correctness. & \\
\hline Q9 & Author: Please check the footnote of Tables 2 and 3 as typeset for correctness. & \\
\hline Q10 & Author: Please check the heading "acknowledgment" as typeset for correctness. & \\
\hline Q11 & Wiley: Please check the "conflict of interest" as typeset for correctness. & \\
\hline Q12 & Author: Please provide the publisher name and year of publication for Ref. 16. & \\
\hline Q13 & Author: If Ref. 23 is not a one-page article please supply the first and last pages. & \\
\hline Q14 & $\begin{array}{l}\text { Author: Ref. } 37 \text { has not been cited in the text. Please indicate where it should be cited; or delete } \\
\text { from the Reference List. }\end{array}$ & \\
\hline
\end{tabular}

